\title{
Article \\ The Origin of the Most Energetic Galactic Cosmic Rays: Supernova Explosions into Massive Star Plasma Winds
}

\author{
Peter L. Biermann ${ }^{1,2,3,4}$, Philipp P. Kronberg ${ }^{5,6}$, Michael L. Allen ${ }^{7}$, Athina Meli ${ }^{8,9}$, Eun-Suk Seo ${ }^{10}$ \\ ${ }^{1}$ MPI for Radioastronomy, Bonn, Germany; plbiermann@mpifr-bonn.mpg.de \\ 2 Dept. Physics., Karlsruher Institut für Technologie, Karlsruhe, Germany \\ ${ }^{3}$ Dept. of Phys. \& Astron., Univ. of Alabama, Tuscaloosa, AL, USA \\ ${ }^{4}$ Dept. of Phys. \& Astron., Univ. of Bonn, Germany \\ ${ }^{5}$ Dept. of Phys., University of Toronto, Canada; philkronberg@gmail.com \\ 6 Visiting Scholar, Theor. Div., MS B285, Los Alamos National Laboratory, Los Alamos, NM 87545, USA \\ ${ }^{7}$ Dept. of Phys. \& Astron., Washington State University, Pullman, WA, USA; mlfa.astro@gmail.com \\ 8 AGO Dept., University of Liège, Belgium; ameli@uliege.be \\ ${ }^{9}$ Dept. of Phys., University of Gent, Belgium \\ ${ }^{10}$ Dept. of Phys., University of Maryland, College Park, MD, USA; Seo@Umd.edu \\ * Correspondence: plbiermann@mpifr-bonn.mpg.de; Tel.: +1-205-348-3797 \\ $\ddagger$ These authors contributed equally to this work.
}

\begin{abstract}
We propose that the high energy Cosmic Ray particles around the spectral turn-down commonly called the knee and up to the upturn, commonly called the ankle, mostly come from Blue Super Giant star explosions. At the upturn, i.e. the ankle, Cosmic Rays probably switch to another source class, most likely extragalactic sources. To show this we recently compiled a set of Radio Supernova data to list the magnetic field, shock speed and radius scale (Biermann et al. 2018) [43]. Using particle acceleration theory at shocks, those numbers can be transformed into characteristic knee and ankle energies. Without adjusting any free parameters both of these observed energies are directly indicated by the supernova data. We now proceed to the next step in the argument, and use the Supernova Remnant data of the starburst galaxy M82. Assuming that they are Blue Supergiant star explosions, the shock will race to their outer edge with a magnetic field that follows $B(r) r \sim$ const.. We argue that the shock runs through the entire magnetic plasma wind region at full speed all the way out to the wind-shell, which is of order parsec scale. The speed is observed to be $\sim 0.1 \mathrm{c}$ at about $10^{16} \mathrm{~cm}$ radius in the plasma wind. This demonstrates how Blue Supergiant star explosions can provide the Cosmic Ray particles across the knee and up to the ankle energy range. The data from the CREAM (Cosmic Ray Energetics and Mass Experiment) mission will test this cosmic ray concept which is reasonably well grounded in two independent radio supernova data sets. The next step in developing our understanding is to obtain accurate Cosmic Ray data near to the knee, and use unstable isotopes of Cosmic Ray nuclei at high energy to probe the "piston" driving the explosion. We plan to combine these data with the physics of the budding black hole which is probably forming in each of these stars to learn more.
\end{abstract}

Keywords: Cosmic Rays; Massive Star Supernovae; Cosmic Ray Knee and Ankle.

\section{Introduction}

Energetic particles with energies far above thermal levels, called cosmic rays, were discovered by Hess (1912) [78] and Kohlhörster (1913) [86], with their energies now known to range up to about $10^{20} \mathrm{ev}$, first seen by Linsley (1963) [100]. It was proposed very early (Baade \& Zwicky 1934) [29] that supernovae could easily provide the energies required for Galactic cosmic rays (GCRs). Ginzburg \& Syrovatskij (1963) [71] suggested that radio galaxies could provide the highest energy component. 
The mechanism of acceleration was originally proposed by Fermi $(1949,1954)$ as repeated reflections between interstellar clouds with magnetic field bottle configurations which are approaching each other. This was later generalized to today's concept of energetic particles being reflected back and forth across a shock wave, separating converging flows (Axford et al. 1977; Krymskii 1977; Bell 1978a,b; Blandford \& Ostriker 1978; [28,32,33,46,95]), with early general overviews by Ginzburg \& Ptuskin (1976) [72] and Drury (1983) [62]. It is now recognized that such particles permeate interstellar space, as well as intergalactic space. Particle energies above order $\mathrm{GeV}$ are found throughout the Solar system. The early arguments on their possible origin were all made on the grounds of sufficient energy supply, and they still stand. However we now know of many other processes and events of stars and black holes that clearly produce energetic particles.

In addition to the supernova origin of accelerated CRs and outflow (the theme of this paper), is energy outflow and particle acceleration due to central supermassive black holes, and their jets and lobes. Cosmic rays, magnetic fields and energy from SMBH-powered jets are additional phenomena to SN- generated CRs. They have been discussed by e.g., Ginzburg \& Syrovatskij (1963) [71], Lovelace (1976) [101], Biermann \& Strittmatter (1987) [35], Kronberg et al.( 2001) [92], Kronberg (2002) [93], Colgate (2004) [56], Colgate et al. (2014) [57], Biermann et al. (2016) [42], and in many other papers.

Some recent relevant reviews and books are Aharonian (2004) [19], Stanev (2010) [133], Kotera \& Olinto (2011) [87], Letessier-Selvon \& Stanev (2011) [99], Bykov et al. (2012) [49], Diehl (2013, 2017) [60,61], Blasi (2013) [47], Gaisser et al. (2016) [68], Kronberg (2016) [94], Amato \& Blasi (2017) [27], and Biermann et al. (2018) [43], referred to as ASR18. Many further tests can now be done with new results from Auger, the Telescope Array, IceCube, AMS, HAWC, HESS, MAGIC, CALET, DAMPE, and Veritas, further balloon flights, such as TIGER, and other experiments in space, on balloons, and on the ground. ISS-CREAM is the next major space experiment with new data yet to come, following on AMS (Seo et al. 2014, Seo 2018, [126,127]).

\section{Massive star plasma winds}

Massive stars explode as Supernovae (SNe). The shock pushed out by the explosion can accelerate cosmic ray $(\mathrm{CR})$ particles. When this shock races through a stellar magnetic plasma wind, particle energies can go as high a few $10^{18} \mathrm{eV}$ (Stanev et al. 1993, referred to as CR-IV, [132]). Massive stars with magnetized plasma winds come in two varieties, Red Supergiant (RSG) stars above about $25 M_{\odot}$ Zero Age Main Sequence (ZAMS) mass, and Blue Supergiant (BSG) stars, above about $33 M_{\odot}$ ZAMS mass. All of these are in binary systems, but not all of them are in tight orbits. RSG star magnetic plasma winds have a low velocity and high density whereas BSG star magnetic plasma winds have a very high velocity and low density. In the time period preceding the $\mathrm{SN}$ explosion, this plasma wind experiences short episodes during which the star may enhance its mass loss, and magnetic fields (as summarized with many references, e.g., in ASR18 [43]). The plasma wind terminates in a shell, where it encounters the interstellar medium (ISM), the molecular cloud (MC), out of which the star formed, and/or the wind-bubble environment of preceding $\mathrm{SN}$ explosions in its immediate environment. As the ram pressure of RSG star plasma winds is two orders of magnitude lower than for BSG star plasma winds, the wind shell may have a radial scale about an order of magnitude smaller than BSG star plasma winds. It also follows that the life-time of activity for RSG star explosions is an order of magnitude shorter than for BSG star explosions. This provides a strong counter selection effect in identifying such sources. When the $\mathrm{SN}$ shock does hit the wind shell, it slows down considerably; for radio supernovae (RSNe) the SN-shock speed is typically 0.1c, while for the Super Nova Remnants (SNRs) in M82 (Kronberg et al. 1985, hereafter referred to as KBS85, [90]) the observed velocities of expansion (e.g. Fenech et al. 2008, 2010, [63,64]) are all far below this value; this observation matches the expectation that upon encountering the wind shell the $\mathrm{SN}$ plasma shock reverts to environment-dominated expansion (e.g. Cox 1972). That reversion causes the shocked region to be about $1 / 4$ of the spherical volume encompassed by the shock, rather than $3 / 4$ as in the shocked region in a wind (Biermann \& Cassinelli 1993, [38], Biermann \& Strom 1993, [39], referred to as CR-II and CR-III; this uses a strong shock, see 
Landau \& Lifshitz 1959, [98], and Drury 1983, [62]). When a SN shock races through a stellar plasma wind, it produces radio emission that can be detected with interferometry (i.e. sub-arcsecond radio imaging) to determine the magnetic field $B(r)$, the mass loss $\dot{M}_{\star}$, its radial scale $r$, and velocity $V_{S N} / c$. We note that $V_{S N}$ is the velocity of the shock in the medium, through which it propagates, here the wind of the star. As these shocks are observed to show about $0.1 c$, we can neglect the prior velocity of the wind. From these three quantities, scale $r$, magnetic field $B$, and shock speed $V_{S N} / c$ we can derive two critical energies for the maximal energies particles can reach in shock acceleration, $E_{\text {knee }}$ and $E_{\text {ankle }}$, first the maximal energy of a particle fitting into the space available (for a plasma simulation see Meli \& Biermann 2006, [105], and for an analytical derivation see, e.g., Biermann 1993, referred to as CR-I, [37], or ASR18 [43]),

$$
E_{\text {ankle }}=\frac{1}{8} \mathrm{ZeB} \mathrm{B}_{0} r_{0}
$$

where $e$ is the elementary charge, $Z$ is the charge of the particle under consideration (cgs units). We have used here the Parker (1958) [121] plasma wind property that

$$
B_{\phi}(r) \times r=B_{0} r_{0} \sim \text { const. }
$$

consistent with the observations discussed here; here $B_{\phi}$ is the dominant component of the magnetic field (Parker 1958, Weber \& Davis 1967 [121,142]). We interpret the associated energy as the ankle in the spectrum of Galactic CRs (GCRs). This corresponds to energies reached in perpendicular shocks (see Jokipii 1987, [82], Völk \& Biermann 1988, [138], CR-I, CR-IV, Meli \& Biermann 2006, [37,105,132], and ASR18 [43]). A lower break energy corresponds to the knee, where the spectrum turns down:

$$
E_{\text {knee }}=\frac{1}{8} \mathrm{Ze} B_{0} r_{0}\left(\frac{V_{S N}}{c}\right)^{2} \text {. }
$$

For the observed numbers of RSNe (ASR18 [43]) the energies derived match the energies for knee and ankle directly, to within the errors, without using any parameter adjustment. We note that to obtain these numbers we take the observed data and average the products $\langle r \times B(r)\rangle$ and $\left\langle r \times B(r) \times\left(V_{S N} / c\right)^{2}\right\rangle$. The numbers show that the ratio of these two averages is $10^{-1.6}$ between them, instead of $10^{-2}$, as would be expected by multiplying separate averages, suggesting the possibility that these numbers might be weakly correlated.

However, as these observations show the situation typically around $r \simeq 10^{16} \mathrm{~cm}$, due to opacity effects, we need to ascertain what happens at the larger scale, when the SN-shock actually hits the wind shell or other environment. This is possible with the data from M82:

We propose to test this concept with the observations of the SNRs in M82 (KBS85 [90]; Bartel et al. 1987, [30]; Muxlow et al. 1994, [117]; Golla et al. 1996, [73]; Allen \& Kronberg 1998, [26]; Allen 1999, [25]; Kronberg et al. 2000, [91]; McDonald et al. 2002, [104]; Muxlow et al. 2005, [118]; Fenech et al. 2008, 2010, [63,64]; Gendre et al. 2013, [69]).

\section{The Super Nova Remnants in the starburst galaxy M82}

Here we show the data on all supernova remnants (SNRs) in M82 that have published derived estimated magnetic fields and for which the size was clearly established (Allen \& Kronberg 1998, Allen $1999,[25,26]$ with many further references therein):

The flux density values listed in Table 1 for the non-thermal emission are referenced to a frequency of $1 \mathrm{GHz}$, and were obtained by a fit across very many frequencies, from $408 \mathrm{MHz}$ all the way to $23.46 \mathrm{GHz}$, using a screen model absorption and separating thermal from non-thermal emission. The spectral index given is that of the non-thermal straight power-law component. The magnetic field derived in Allen \& Kronberg (1998) [26] has to be scaled with the factor $\{(1+k) / \phi\}^{2 / 7}$, which is highly uncertain; here $k$ is the ratio of the CR proton energy density to that of CR electrons, at an energy of a few $\mathrm{GeV}$ - at lower particle energy this ratio is expected to be smaller, since protons do not 
Table 1. Supernova remnants (SNRs) in starburst galaxy M82, based on Allen \& Kronberg (their Table $5 ; 1998)$ [26], in turn based on Allen (1999) [25]

\begin{tabular}{|c|c|c|c|c|c|}
\hline Coordinate name & $\begin{array}{c}\text { size } 2 r \\
\text { in pc }\end{array}$ & $\begin{array}{c}\text { flux density } \\
\text { in mJy }\end{array}$ & sp. index & $\begin{array}{c}\text { est. magnetic field } \\
B \text { in mGauss }\end{array}$ & $\begin{array}{c}\log (B r) \\
\text { in Gauss } \times \text { cm }\end{array}$ \\
\hline $40.68+550$ & 3.72 & 17.9 & -0.52 & 1.80 & 16.0 \\
$41.31+596$ & 2.17 & 8.59 & -0.54 & 2.32 & 15.9 \\
$41.96+574$ & 0.33 & 122.8 & -0.72 & 26.4 & 16.1 \\
\hline $42.53+619$ & 1.71 & 30.9 & -1.84 & 11.7 & 16.5 \\
$42.67+556$ & 3.02 & 4.44 & -0.61 & 1.46 & 15.8 \\
$43.19+583$ & 1.16 & 15.3 & -0.67 & 4.79 & 15.9 \\
\hline $43.31+591$ & 3.02 & 30.3 & -0.64 & 2.54 & 16.1 \\
$44.01+595$ & 0.78 & 62.0 & -0.51 & 9.83 & 16.1 \\
$44.52+581$ & 3.72 & 7.2 & -0.61 & 1.40 & 16.9 \\
\hline $45.18+612$ & 3.49 & 24.1 & -0.68 & 2.13 & 15.8 \\
$45.86+640$ & 1.09 & 4.10 & -0.53 & 3.39 & 16.0 \\
$46.52+638$ & 3.88 & 9.71 & -0.73 & 1.53 & 15.9 \\
\hline $46.70+670$ & 3.4119 & 5.22 & -0.57 & 1.37 & \\
\hline
\end{tabular}

contribute any extra energy below their rest mass energy, but electrons continue to contribute due to their smaller rest mass. From above we adopt $\phi=1 / 4$, since clearly the SN-shock has encountered the outer shell of the wind, and is observed to have slowed down. We list here the magnetic field without any such correction.

\subsection{The $C R$ proton/electron ratio $k$}

For the $\mathrm{CR}$ proton/electron ratio $k$, observed to be about 100 above a few $\mathrm{GeV}$, we can make the following argument, based on the observations of young RSNe (see ASR18 [43]): The first process that happens in an electron-proton shock is the thermalization of these particles. Thermalization has to work at all radii, from below $10^{16} \mathrm{~cm}$ out to parsec scale, rendering the density lower by a factor of $10^{5}$ at least. The wind is magnetic with the basic configuration of the magnetic field a Parker type spiral, so essentially yielding a perpendicular shock configuration. This process has been discussed in Spitzer (1962) [131], but his treatment is non-relativistic and uses isotropic phase space distributions; then simple electron-proton scattering is far too slow at high temperature. When the protons go through the shock, they fill in phase space a torus with the radius in momentum $m_{p} V_{S N}$, and with the relative width of the inverse of the Mach-number, so corresponding to the upstream speed of sound. That entails that this anisotropic momentum distribution causes turbulence at all the scales from the maximum on down, over a range of the Mach-number. But because the Mach-number is close to $m_{p} / m_{e}$ the smallest scales in this range correspond to the waves matching the scales of the electrons with the maximal momentum $m_{e} V_{S N}$. This means that at all these scales the protons and electrons are scattered in momentum phase space. We have argued in ASR18 [43] that the spectrum of irregularities in the direct shock region is $I(k) k \sim 1$ and so the power per log bin of wave-number is equal at all scales. It follows that thermalization occurs. Due to all this turbulence the time scale of thermalization is of order a small multiple of the thermal Larmor radius divided by the Alfén speed, so highly adequate, by a factor of $2 E_{k n e e} /\left(m_{p} V_{S N}^{2}\right)$, and a fortiori $2 E_{\text {ankle }} /\left(m_{p} V_{S N}^{2}\right)$. The time scale for electrons might be $m_{p} / m_{e}$ times slower due to the anisotropy of the scattering, but that is still quite adequate by many powers of ten. This time scale scales with the radius, and so works the same at all relevant radii, the condition noted above.

Thermalization yields a first characteristic energy of the electrons, $\gamma_{e, s h} c m_{e}$. Beyond this energy, our interpretation of the radio observations (ASR18 [43]) then says that the electrons gain energy only by drifts (CR-I [37]); shock acceleration does not work yet, because the electrons do not see the shock. In ASR18 [43] we noted that the electrons so only get stochastic shock drift acceleration, giving them a spectrum of -3.06 , from observations, say about -3 (this line was reasoning was also used for ions by 
Caprioli 2012 [51]). This drift dominated spectrum ranges to that momentum, at which the energetic electron reach a Larmor radius that equals that of the shock thermal protons, $\gamma_{s h, i n j} c m_{e}$, (i.e., the electrons have the same Larmor radius as shock-protons; if another $\mathrm{CR}$ ion is dominant, corresponding to the Larmor radius of that ion, with mass number $A_{\text {dom }}$ and charge at injection $Z_{d o m}$ ). From this second momentum electrons "see" the shock, and have the normal CR accelerated spectrum, say $-7 / 3$ for the most common CR component of CRs accelerated in a wind (see ASR18 [43]). Protons attain the maximal energy content only at a few $\mathrm{GeV}$ (i.e. just above their rest mass energy). This applies to any particle number spectrum even slightly steeper than -2 . That implies that electrons have a higher energy content at that momentum, where the still are just above thermal in the post-shocked region. We assume that at this stage that electrons are in equipartition with the protons (or dominant ions), at the start due to charge neutrality.

This means that

$$
\gamma_{e, s h} c^{2} m_{e}=\frac{1}{2} A_{d o m} m_{p} c^{2} \beta_{s h}^{2}
$$

and

$$
\gamma_{s h, i n j} c m_{e}=\frac{A_{d o m}}{Z_{d o m}} m_{p} c \beta_{s h}
$$

So between these two energies the energy density of CR electrons runs down by a factor of

$$
\frac{2}{\beta_{\text {sh }}} \frac{1}{Z_{d o m}}
$$

in the first step. Here we use the observations of a spectral index of about $E^{-3}$ implying that the energy content above a given energy runs as $E^{-1}$, so here giving a factor of about 20, with the observed $\beta_{\text {sh }}=0.1$ (see ASR18 [43]).

There is an observational check on this, as the electron energies required to explain the radio observations of RSNe need to be above that first energy: We observe the emission at a frequency of 5 $\mathrm{GHz}$, at a magnetic field of order Gauss requiring a Lorentz factor of $\gamma_{e} \simeq 30$, while $\gamma_{e, s h}$ for protons is about 10, so well below this. However, this argument implies that the emission should have a cutoff on the low frequency side, perhaps visible somewhat early in the emission phase and at lower frequency, such as $400 \mathrm{MHz}$. It also implies that at that stage of the emission there are constraints for what ions could possibly dominate to explain the emission, implying that in this numerical example $A_{\text {dom }} \lesssim 3$, eliminating basically all common elements other than Hydrogen.

In the second step, using a spectrum of $p^{-7 / 3}$ (see ASR18 [43]) the electrons reduce their energy in that range, where the protons do not contribute anything. Being below relativistic, so up to the energy of the proton rest mass (or dominant ion again) the factor is

$$
\left(\frac{Z_{d o m}}{\beta_{s h}}\right)^{+1 / 3}
$$

which is about 2 , using the same data again, with protons. So the CR electron energy density is reduced by a combined factor of $k \simeq 40$ when their momentum reaches that of the relativistic protons, where this factor is determined by observations. Already Allen \& Kronberg (1998) [26] mentioned 40 as a possible value for $k$.

The argument makes it obvious that other sources, for which $\beta_{s h}$ is smaller, contribute even less. That in turn implies that the combination of various sources contributing to electrons and protons reduces the electron net contribution even more, down to the observed factor of 1 in 100 . All this starts with the energetic electrons having exactly the same energy density as the dominant ions in the shock, due to charge conservation. To the degree that protons have a strong contribution from ISM-SN-CRs, which have a slightly steeper spectrum than the most common wind-SN-CR component as well as 
slower shocks, this suggests that at moderate energies the CR electrons have a spectrum slightly flatter than protons.

\subsection{The magnetic field in the $S N$-shocked wind, and the implications}

All this suggests that $k=40$ is appropriate; covering this range of added assumptions implies an uncertainty of order $10^{ \pm 0.2}$. This leads to a correction factor of 4.29 over the magnetic field values listed in Allen \& Kronberg (1998) [26], and repeated above. However as we argued above, that the shock must have slowed down significantly already from its free expansion phase through the wind, and used the ISM-Sedov volume fraction of the shocked shell of $1 / 4$, we need to recognize that the magnetic field is likely to have been squashed by a new reverse shock pushed back through the material, giving the magnetic field an extra factor of 4, effectively cancelling the factor of 4.29. The magnetic field which we wanted to test was the value before any additional enhancement, still in the pure wind-shock mode; therefore we should reduce the magnetic field value obtained by a factor of 4 , coming back to the original numbers by Allen \& Kronberg (1998) [26]. This supports the argument by Allen \& Kronberg (1998) [26] to give their magnetic field value without such an uncertain correction.

The average of the last column is 16.0: Using the calibration from Allen \& Kronberg (1998) [26] and their flux density values we can also estimate the corresponding value of $\langle\log \{B \times r\}>$ from the table in Fenech et al. (2010) [64], who did not include a separation of thermal and non-thermal emission, nor any absorption. However, to within the errors, we obtain the same number with a larger scatter confirming the analysis done by Allen \& Kronberg (1998) [26]. Using the specific numbers here, a factor of $10^{0.1}$ higher than in ASR18 [43], gives for the ankle energy

$$
E_{\text {ankle }}=\mathrm{Z} 10^{17.6 \pm 0.2} \mathrm{eV},
$$

with the larger error due to the systematics, and now using $\mathrm{eV}$ for energy as is custom in the $\mathrm{CR}$ community.

Observations of the CR spectrum, mass composition and arrival direction anisotropy between knee and ankle, and to higher energies above the ankle, such as in Aab et al. (2017a, b, 2018) [1-3], Abbasi et al. (2018a, b, c) [8-10] and Abeysekara et al. (2018a) [14] will allow us to discern the details of highest energy tails of GCRs and the transition to extragalactic CRs.

Two questions remain, (i) whether the plasma wind maintains its magnetic profile $B_{\phi} \sim r^{-1}$ (Parker 1958, Weber \& Davis 1967, [121,142]), and (ii) whether the SN-shock slows down. We have shown in ASR18 [43] from simple estimates of the ram-pressure in RSG star winds, and BSG star winds, that a RSG wind shell may be an order of magnitude smaller than a BSG star wind shell. That difference entails (see for a detailed estimate ASR18 [43]) that both in RSG star winds as in BSG star winds the SN-shock has the chance to race all the way to the wind shell, before converting into a more Cox-like Sedov expansion (Cox 1972 [58]; a Sedov expansion implies conservation of energy, Sedov 1958, [125]) through the wind shell and outer medium; the sparse data on velocities (e.g. Fenech et al. $2008,2010[63,64])$ show that all measured velocities are below $0.1 c$, usually far below. In the data listed above in Table 1 there is no hint of any correlation between size and spectral index. Just from simple mass function arguments, the ratio of RSG star explosions to BSG star explosions is expected to be of order 1 to 2; however, in RSG star wind shells the density is far higher than in BSG star wind shells. Therefore the cooling is much more efficient. This implies that we have two development phases: First we have the racing phase, when the SN-shock goes through the plasma wind; for RSG star winds we may have an intermediate wind-Sedov phase (see ASR18 [43]); then we have the cooling phase, when the shock hits the outer shell, and the shock region transforms rapidly from a Sedov-like shock to a cooling shock. Both these stages for RSG star explosions are much shorter than for BSG star explosions. Consistently with such an expectation, in these data the typical size is about $1 \mathrm{pc}$, with only 1 source at about $0.1 p c$, which may be a RSG star explosion, or a BSG star explosion in a very high pressure environment. 
In ASR18 [43] we also derived the required piston mass to keep driving the shock; the number we obtained from $\gamma$-ray line observations was about $0.1 M_{\odot}$. The question is whether this could be the ${ }^{56} \mathrm{Ni}$ mass derived from optical observations (Maeda et al. 2010; Hamuy 2003; Utrobin et al. 2017; Nakar et al. 2016; Lusk \& Baron 2017, [75,102,103,119,134]). Extrapolating from lower mass stars this seems possible, however, the numbers derived are highly model dependent, so for instance adopt usually 1D modelling rather then 3D modelling (for a 3D model see Utrobin et al. 2017) [134], so plumes of heavy elements cannot easily be accounted for (e.g., Biermann et al. 1992 [36], Wesson et al. 2015 [144]): Using just the energy in the ${ }^{56} \mathrm{Ni}$ decay chain $\left({ }^{56} \mathrm{Ni}->{ }^{56} \mathrm{Co}->{ }^{56} \mathrm{Fe}\right.$, e.g., Lusk \& Baron 2017 [102]) should allow reliable numbers barring any issues with radiation transfer. We note (following again ASR18 [43]) that the mass suggested by the $\gamma$-ray line observation is certainly sufficient to drive a SN-shock all the way out to parsec scale radial distances in BSG star plasma winds, and do so at maximal velocity: the mass accumulated in a BSG star wind can be written as

$$
\Delta M_{B S G, \text { wind }}=10^{-2.3} M_{\odot} \frac{\dot{M}_{\star-5}}{V_{W, 8.3}} \frac{r}{\mathrm{pc}^{\prime}}
$$

where $\dot{M}_{\star-5}$ is the mass loss of the star prior to the explosion in units of $10^{-5} M_{\odot} \mathrm{yr}^{-1}$, and $V_{W, 8.3}$ is its wind velocity in units of $2000 \mathrm{~km} / \mathrm{s}$. The mass loss is directly determined in modelling the radio observations of the radio supernovae (ASR18 [43] and all its references in its Tables 1 and 2), and the wind velocity is typical for such stars. This shows that a piston of $0.1 M_{\odot}$ could drive the SN-shock unimpeded to scales of order 10 parsec, as long as the ram pressure is sufficient to overcome the environment.

This allows another consistency test of our concept and its numbers: The kinetic energy should exceed the magnetic field energy significantly. A piston of $0.1 M_{\odot}$ at $0.1 c$ corresponds to $10^{51} \mathrm{erg}$, while the typical magnetic field strength of about $\simeq 5$ mGauss corresponds to $10^{49.5} \mathrm{erg}<<10^{51} \mathrm{erg}$, using the volume fraction of $1 / 4$ adopted above.

For the ram pressure we need to consider first the wind ram pressure against the outside medium, and then the $\mathrm{SN}$-shock ram pressure. The wind ram pressure condition for matching the ISM pressure is

$$
r<30 \text { pc } \dot{M}_{\star-5}^{1 / 2} V_{W, 8.3}^{1 / 2} P_{I S M,-12}^{-1 / 2}
$$

where $P_{I S M,-12}$ is the outside pressure in units of $10^{-12} \mathrm{dyn} \mathrm{cm}^{-2}$, a typical number for the outside pressure in our Galaxy. The higher pressure in M82 would allow smaller sizes for the wind shell, but of course the local pressure where the SNRs are seen, may be lower than in the central region. A pressure $10^{3}$ times higher would still allow the size scales observed for the SNRs in M82. The region where the SNRs are seen has been described as a ring of about 500 pc radius (Weliachew et al. 1984, Kronberg \& Wilkinson 1975, and Kronberg et al. 1981, [88,89,143]). Inside that ring the pressure might be even higher. If the ISM-pressure inside that ring were two orders of magnitude higher, for instance, as argued in KBS85, then the maximal size would only be 3 pc, still somewhat larger than the SNRs seen.

The corresponding $\mathrm{SN}$-shock ram pressure condition is

$$
r<10^{2.7} \text { pc } \dot{M}_{\star-5}^{1 / 2} V_{W, 8.3}^{-1 / 2} V_{S N,-1} P_{I S M,-12}^{-1 / 2}
$$

where $V_{S N,-1}$ is the shock speed in units of $0.1 \mathrm{c}$. However, we also need to double-check when the mass accumulated outside within the extra radial range $\Delta r$ slows down the progression of the shock, and that is given by

$$
\left(\frac{\Delta r}{r}\right)=10^{-0.7} M_{p i s t,-1} r_{p c}^{-3} n^{-1}
$$

where $M_{\text {pist, }-1}$ is the mass of the piston in units of $0.1 M_{\odot}, r_{p c}$ is the radius in pc when the SN-shock hits the outer medium, and $n$ is the outside medium density in units of $\mathrm{cm}^{-3}$. That implies that 
once the SN-shock hits the wind shell and outside medium at full speed, the shock slows almost immediately to the Sedov stage, and only shortly later to the cooling phase. Adding in the mass accumulated in the wind does not modify this conclusion significantly. Nothing earlier slows down the SN-shock in a BSG star wind.

It follows that the knee energy predicted here is

$$
E_{\text {knee }}=\mathrm{Z} 10^{16.0 \pm 0.2} \mathrm{Z} \mathrm{eV},
$$

We conclude that to within the errors these numbers on the product of radial scale and magnetic field in the table 1 and in tables 1 and 2 in ASR18 [43] are the same, supporting our argument, that in these examples the $\mathrm{SN}$-shock pushed out without losing significant strength, and so maintaining these energies. Also, all of these examples in M82 are consistent with being BSG star explosions. All of the SNRs seen in M82 are consistent with being in the slow-down phase, after the SN-shock has hit the wind shell or other environment.

\section{The origin of high energy Galactic cosmic rays}

As we discussed and have shown above: RSG star explosions produce high energy (HE) Galactic cosmic rays (GCRs), i.e. energies around the knee, with a near normal abundances but not to the highest energies, and BSG star explosions give HE GCRs with all heavy elements enhanced and to the highest energies for GCRs. If our simple picture, deduced above, is correct, that RSG star explosions just terminate earlier, at a radius about 1/10 of that of BSG star explosions; the anti-proton data (ASR18 [43]) in our Galaxy suggest, that the RSG star explosions run their shock down in velocity, while the BSG star explosions hit their respective wind shell at full speed. We note that this distinction may be different in the galaxy M82, due to the higher environmental pressure. Therefore the characteristic energies, $E_{\text {knee }}$ and $E_{\text {ankle }}$ are maintained only for the BSG type of explosion. It ensues that at the highest CR energies, RSG star explosions contribute less than the BSG star explosions. Since in our model these energies scale with nuclear charge number $Z$, at a down-turn of the spectrum, near the knee the heavier elements go into dominance (see CR-IV [132]), and at the final cutoff, at the ankle energy, protons will disappear very much earlier than Carbon, Oxygen or heavier elements such as Iron (see Fig 6 in CR-IV, as well as Fig.1 in ASR18 [43,132]). We emphasize again, that the observed characteristic energies $E_{\text {knee }}$ and $E_{\text {ankle }}$ are directly reproduced by two independent data sets on RSNe and SNRs. This is based on RSNe in other galaxies, and SNRs in the starburst galaxies M82, without invoking any special parameter to be adjusted. The two data sets agree well within the errors. The spectrum both below and above the knee, can be explained with a combination of Diffusive Shock Acceleration (DSA) and Stochastic Shock Drift Acceleration (StSDA), as shown first in CR-I, CR-II, CR-III, and CR-IV, rederived in ASR18 [37-39,43,132], all based on earlier work by Drury (1983), Jokipii (1987), Völk \& Biermann (1988), [62,82,138] and others as cited in CR-I, and ASR18 [37,43]. One consequence is that most interaction happens in the wind-shell, when the SN-driven shock hits (e.g. Biermann et al. 2001 [40]). In ASR18 [43] we discussed a number of consequences, that allow this concept to be tested with the spectra of secondary particles. Here we just note that the concept entails that the $\gamma$-ray -spectra of the Galaxy should reflect this early interaction, showing evidence for a spectrum of mostly $E^{-7 / 3}$ from the $4 \pi$ component in wind-SN-CRs, and a weaker polar cap component of $E^{-2}$ : A thorough analysis of the Fermi data already demonstrates that the data require two such spectral components (de Boer et al. 2017 [48]). HAWC (High Altitude Water Cherenkov Observatory) and HESS (High Energy Stereoscopic System) data are fully consistent with this prediction of a dominant $4 \pi$ component (e.g. Nayerhoda et al. 2018 [120]). At higher $\gamma$-ray energies the spectrum produced is predicted to become flatter due to the emergence of the polar-cap component with $E^{-2}$ in wind-SN-CRs (CR-IV [132]). Other new data, e.g. from AMS (Alpha Magnetic Spectrometer on the ISS) or HAWC, such as the spectra of various elements and secondary and primary contributions to Ni in cosmic rays (ASR18, Alfaro et al. 2017, Aguilar et al. 2017, 2018a, b [16-18,23,43]) can also be used as further tests.

However, there are a number of unanswered questions with this simple picture: 
1) Very rarely we do have Gamma Ray Bursts (GBRs) in any star forming galaxy, at a rate of order $10^{-2}$ of massive stars. There is no question again, that they accelerate particles to high energy. Where is their contribution from GRBs in our own Galaxy? Could they contribute also below the ankle? Or is this already part of the extragalactic contribution, as argued in many papers, such as Milgrom \& Usov (1995, 1996) [108,109]; Vietri (1995, 1996, 1998) [135-137]; Waxman (1995), Miralda-Escudé \& Waxman (1996), Waxman \& Bahcall (1997) [115,140,141]; Zhang \& Mészáros (2004) [146]; Piran (2004) [122]. In ASR18 [43] we proposed that the compact radio source 41.9+58 in the starburst galaxy M82 (KBS85 [90]) is in fact the result of a recent merger of two stellar black holes (BHs), with a concurrent GRB (see Muxlow et al. 2005 [118], who argued first for a misaligned GRB for this source). The argument then proceeds to propose that this BH merger could explain the Ultra High Energy CR (UHECR) particles detected by the Telescope Array (TA) detector (Abbasi et al. 2014, 2018a [7,8]; and as cited in Biermann et al. 2016 [42]). Statistically GRBs do not seem to contribute much to UHECRs (Meli et al. 2008, Abbasi et al. 2012, Albert et al. 2017a, [6,20,106]). Meli et al. (2008) [106] also argued that GRBs in simulations and using a diffuse model do not contribute significantly to the UHECR population. All this depends on the model assumptions, and so may not be in contradiction.

2) There are many arguments in support of the sources of UHECRs from radio galaxies (e.g., Ginzburg \& Syrovatskij 1963, Hillas 1984, Biermann \& Strittmatter 1987, Meli et al. 2008, [35,71,80,106]), with a special interest in the case, when their relativistic jets are pointed at us, visible in the form of flat-spectrum-radio quasars (FSRQs) or blazars (Kadler et al. 2016; Kun et al. 2017, 2018; Aartsen et al. $2018 \mathrm{a}, \mathrm{b},[4,5,83,96,97])$ : There are now a total of nine candidate identifications for high energy neutrino events with FSRQs (see also the lectures of F. Halzen at Erice 2018 [74]), many of them suggestive of an ongoing merger of two super-massive black holes (Gergely \& Biermann 2009, Caramete \& Biermann 2010, Kun et al. 2017, [52,70,96]). One of these candidates, TXS0506+056 is the most convincing, since it shows evidence for several neutrino events. To make HE neutrinos requires the acceleration of $\mathrm{HE}$ and plausibly UHECR particles first, so this tentative finding allows the speculation that mergers of super-massive black holes may lead to ubiquitous production of UHECR particles. This entails that UHECR production is highly episodic. Do these sources also contribute below the ankle?

3) These BSG explosions can yield the energy, but can the CRs produced actually reach us? This is especially an issue for electrons and positrons: in ASR18 we argued that we need electrons up to $30 \mathrm{TeV}$ in the source region; HESS has now detected electrons at $20 \mathrm{TeV}$, so far published only on its website [79], and in conference proceedings. The CR electron spectrum shown on the HESS website is consistent with our 2009 prediction (Biermann et al. 2009 [41]) of at lower energies first $E^{-10 / 3}$, then $E^{-3}$, and last, at the highest energies, $E^{-4}$, all based on the idea that many sources contribute under lots of losses (Kardashev 1962 [84]); at the highest energies the observed spectrum is slightly flatter than $E^{-4}$, possibly due to local and/or recent nearby sources. We note that the synchrotron loss time of electrons at $20 \mathrm{TeV}$ is of order $3 \cdot 10^{4} \mathrm{yrs}$ (using a magnetic field strength of order $5 \mu$ Gauss: Beck et al. 1996 [31]; a crude estimate of $5 \mu$ Gauss was first made in the 1940s), implying a source rather close by (as has been argued by many, e.g., Yüksel et al. 2009 [145]), or a very anisotropic transport to allow larger distances; an alternate way of phrasing this speculation could be that the magnetic fields are extremely structured allowing some transport over large distances.

4) The relative contribution of CR particles from RSG star explosions and BSG star explosions at low energy is about 4:1, following Binns et al. (e.g. Murphy et al. 2016 [116]); what is it at high energy? The anti-proton data suggest, that the RSG star explosions shocks run out of steam, and so that RSG star explosions in our Galaxy contribute much less at the maximal energies than the BSG star explosions. This may imply that the piston mass in RSG star explosions is smaller than in BSG star explosions, consistent with a naive interpretation of the ${ }^{56} \mathrm{Ni}$ data:

5) Can the ${ }^{56} \mathrm{Ni}$ mass derived from optical observations (Hamuy et al. 2003, Maeda et al. 2010, Nakar et al. 2016, Lusk \& Baron, 2017, Utrobin et al. 2017, [75,102,103,119,134]) be identified with the piston? The numbers seem to match rather well, but have not been derived for BSG star explosions, and rely mostly on rather simple 1D models, as we discussed above, and of course on using the bolometric 
light-curve; and in fact, the numbers suggest a lower mass for RSG stars, as speculated above. We need to emphasize that we do not actually know the explosion mechanism for very massive stars, be it due to neutrinos (e.g. Bethe 1990 [34]), magnetic fields (e.g. Bisnovatyi-Kogan 1970, Bisnovatyi-Kogan et al. 2008 [44,45]), or - a newly worked out suggestion - quark deconfinement (Fischer et al. 2018 [67]) as the engine.

6) Do SN Ia explosions contribute anything to the observed CR particle population? The $\mathrm{D}^{6}$ scenario seems to be favored now (Shen et al. 2018a [129]; $D^{6}$ here stands for Dynamically Driven Double-degenerate Double-detonation), especially with now also identifying some dwarf stars remaining from SN Ia explosions (Shen et al. 2018b, Raddi et al. 2018 [123,130]) in the Gaia data. The radio data (e.g. Dickel et al. 1991 [59]) and the interpretation of the radio and X-ray data (e.g. Chevalier 1982 [53]) demonstrate unequivocally that these explosions do accelerate energetic particles (Bykov et al. 2018 [50]). But what do they contribute to what is observed?

7) Pulsars and pulsar wind nebulae are directly observed to accelerate particles to high energy, and for one there is even a possible hint of neutrinos (TeVPA 2018 IceCube lectures; e.g. Aliu et al. 2014 [24]). What is their contribution? The high energy gamma data data clearly demonstrate that they produce energetic particles very significantly, but they cannot contribute easily to the observed positrons (Yüksel et al. 2009; Abeysekara et al. 2017 [13,145]).

8) Relativistic SNe: this is amply discussed in Bykov et al. 2018 [50], in ASR18, and in many earlier papers cited therein. They surely contribute, as they are a variant of GRBs; however, is their contribution mostly at the very highest energies, and what ratio to GRB contributions do they show?

9) Active single and binary stars: Any massive star with a powerful wind contributes (Seo et al. 2018 [128]). Microquasars are binary stars, as are all massive stars (Chini et al. 2012, 2013 [54,55]). Micro-quasars surely produce energetic particles and cosmic rays (e.g. Heinz \& Sunyaev 2002, Mirabel $2004,2011[77,110,111])$. So all of the final evolutionary stages will be active contributors to energetic particles (e.g. Vulic et al. 2018, Abeysekara et al. 2018b [15,139]). But is their contribution discernible in the $\mathrm{CR}$ data?

10) Neutron star and stellar mass BH mergers; as this may lead to a GRB, as observed, they clearly can contribute (Abbott et al. 2017a, b; Albert et al. 2017b [11,12,21]).

11) Transport out of the Galaxy? Radio data allowed Heesen et al. (2018) [76], and Krause et al. (2018) [85] to show that CR transport is typically convective, i.e. in a galactic plasma wind. They give a threshold condition in the form of star formation rate per area (see also Rossa \& Dettmar 2003 [124]). This confirms some very old ideas, going back to Weber \& Davis (1967) [142], Parker (1958) [121] and even earlier notions in the late 1940s.

12) A CR contribution from the wind-shock of the Galactic plasma wind? This has been explored repeatedly (Jokipii \& Morfill 1987 [81]), most recently by Merten et al. (2018) [107]; they find that such a contribution is possible.

13) Neutrino production in the Galaxy? The IceCube and Antares observations begin to constrain the models (Albert et al. 2018 [22]). The $\gamma$-ray data already suggest plausible models, all consistent with the concept here, that interaction is dominant when the SN-shock hits the wind-shell. Analogously to our comments on diffuse $\gamma$-ray emission ensues that there should be two underlying spectra of neutrinos, one at $E^{-7 / 3}$ from the $4 \pi$ component, and at higher energy a lower $E^{-2}$ from the polar-cap component (CR-IV [132]): These emission spectra have a transition at an energy which is not well determined right now. At TeV energies we cannot easily separate the RSG and BSG contributions, where the BSG star contributions may or may not dominate the neutrino emission. The stronger interaction in the RSG star explosions leading to anti-protons in our model proposed may push the transition energy to a higher value in the observations summed over a line of sight.

The overall diffuse Galactic neutrino emission is predicted to show a sharp turn-off of both components near $1 / 20$ of the knee energy for protons, so here

$$
E_{v o f f}=10^{14.7 \pm 0.2} \mathrm{eV},
$$


IceCube will surely be able to test this prediction in the future.

14) What is the explosion mechanism, and do we obtain a BH every time, or most of the time, when BSG stars explode? What is the nature of the piston? The innermost layers that get ejected come from a zone just outside the budding black hole, may reflect on the situation there, as regards density, pressure, E.O.S., and magnetic fields. Are there some stars that produce a black hole without any significant explosion at all (see Mirabel 2017a, b, c [112-114])? Do we have a chance to learn about BH physics from these explosions?

\section{Summary}

Our main task that we set ourselves was to check whether the characteristic energies implied by the numbers for radius, magnetic field, and shock velocity deduced from Radio Super Nova (RSN) data (listed in ASR18 [43]) can be confirmed with the observations of the compact sources, interpreted as Super Nova Remnants (SNRs) in the starburst galaxy M82 (KBS85, Allen \& Kronberg 1998, Allen 1999, $[25,26,90]$ as well as earlier and later references): The answer is positive: The energies for knee and ankle seen in the Cosmic Ray (CR) data can be derived directly, without any free parameter, from these data. That supports the interpretation, that the observed CRs in the energy range from just below the knee all the way to the ankle derive directly from Blue Super Giant (BSG) star explosions. As BSG star explosions are suspected to produce black holes, and to drive the piston pushing out the shock giving rise to $\mathrm{CRs}$, can we learn from $\mathrm{CRs}$ and their detailed properties about $\mathrm{BH}$ formation?

Author Contributions: This work is based on research by M.L.A. with P.P.K.; A.M. covered the plasma physics aspects, and E.-S.S. the space aspects. P.L.B. wrote it up with lots of input.

"This research received no external funding"

Acknowledgments: PLB wishes to thank N. Barghouty, J. Becker Tjus, W.R. Binns, W. de Boer, R. Buta, L. Caramete, S. Casanova, A. Chieffi, R. Chini, R. Diehl, R. Engel, I. Gebauer, L.Á. Gergely, E. Haug, A. Jones, E. Kun, B.B. Nath, V. Ptuskin, F. Sardei, P. Sokolsky, T. Stanev, D. Townsley, and many others for intense discussions of these topics; P.L. Biermann is a member of the Pierre Auger Coll., and as the JEM-EUSO-Coll.; E.-S. Seo is a member of the AMS coll., the CREAM-Coll. and the ISS-CREAM Coll..

Conflicts of Interest: "The authors declare no conflict of interest."

\section{References}

1. Aab, A., Abreu, P., Aglietta, M., et al. (Auger-Coll., includ. P.L. Biermann), (2017a), Title: Combined fit of spectrum and composition data as measured by the Pierre Auger Observatory, Journ. of Cosm. and Astrop. Phys. 04, id.038

2. Aab, A., Abreu, P., Aglietta, M., et al. (Auger-Coll., includ. P.L. Biermann), (2017b), Title: Inferences on mass composition and tests of hadronic interactions from 0.3 to $100 \mathrm{EeV}$ using the water-Cherenkov detectors of the Pierre Auger Observatory, Phys. Rev. D 96, id.122003

3. The Pierre Auger Collaboration: Aab, A., Abreu, P., Aglietta, M., et al. (includ. P.L. Biermann), (2018), Title: Large-scale cosmic-ray anisotropies above $4 \mathrm{EeV}$ measured by the Pierre Auger Observatory, eprint arXiv: 1808.03579

4. IceCube Collaboration: Aartsen, M.G., Ackermann, M., Adams, J., et al. (includ. J. Becker Tjus)), (2018a), Title: Neutrino emission from the direction of the blazar TXS 0506+056 prior to the IceCube-170922A alert, Science 361, 147 - 151

5. IceCube Collaboration: Aartsen, M.G., Ackermann, M., Adams, J., et al. (includ. J. Becker Tjus), (2018b), Title: Multimessenger observations of a flaring blazar coincident with high-energy neutrino IceCube-170922A, Science 361, id. eaat 1378

6. Abbasi, R., Abdou, Y., Abu-Zayyad, T., et al. (IceCube Collab., includ. J.K. Becker Tjus), (2012), Title: An absence of neutrinos associated with cosmic-ray acceleration in $\gamma$-ray bursts, Nature 484, 351 - 354

7. Abbasi, R.U., Abe, M., Abu-Zayyad, T., et al. (Telescope Array Collab.), (2014), Title: Indications of intermediate-scale anisotropy of cosmic rays with energies greater than $57 \mathrm{EeV}$ in the Northern sky measured with the surface detector of the Telescope Array experiment, Astrophys. J. Letters 790, id.L21 
8. Abbasi, R.U., Abe, M., Abu-Zayyad, T., et al. (Telescope Array Collab.), (2018a), Title: Evidence of Intermediate-scale Energy Spectrum Anisotropy of Cosmic Rays $E \geq 10^{19.2} \mathrm{eV}$ with the Telescope Array Surface Detector, Astrophys. J. 862, id.91

9. Abbasi, R.U., Abe, M., Abu-Zayyad, T., et al. (Telescope Array Collab.), (2018b), Title: The Cosmic Ray Energy Spectrum between $2 \mathrm{PeV}$ and $2 \mathrm{EeV}$ Observed with the TALE Detector in Monocular Mode, Astrophys. J. 865, id.74

10. Telescope Array Collaboration: Abbasi, R.U., Abe, M., Abu-Zayyad, T., et al., (2018c); Title: Mass composition of ultra-high-energy cosmic rays with the Telescope Array Surface Detector Data, eprint arXiv:1808.03680

11. Abbott, B.P., Abbott, R., Abbott, T.D., et al. (LIGO-Collab., includ. L. Gergely), IceCube-Collab. (includ. J.Becker Tjus, and T. Stanev), Antares-Collab. (includ. L. Caramete), Integral-Collab. (includ. R. Diehl), Auger-Collab. (includ. P.L. Biermann), and other collaboration teams, (2017a), Title: Multi-messenger Observations of a Binary Neutron Star Merger, Astrophys. J. Letters 848, id.L12

12. Abbott, B.P., Abbott, R., Abbott, T.D., et al. (LIGO-Collab., includ. L. Gergely), (2017b), Title: GW170817: Observation of gravitational waves from a binary neutron star inspiral, Phys. Rev. Letters 119, id.161101, arXiv:1710.05832

13. Abeysekara, A.U., Albert, A., Alfaro, R., et al. (HAWC-Coll, includ. S. Casanova), (2017), Title: Extended gamma-ray sources around pulsars constrain the origin of the positron flux at Earth, Science 358, 911 - 914

14. Abeysekara, A.U., Alfaro, R., Alvarez, C., et al. (HAWC-Coll., includ. S. Casanova),(2018a), Title: Observation of Anisotropy of TeV Cosmic Rays with Two Years of HAWC, Astrophys. J. 865, id.57

15. HAWC Collaboration: Abeysekara, A.U., Albert, A., Alfaro, R., et al. (includ. S. Casanova), (2018b), Title: Very high energy particle acceleration powered by the jets of the microquasar SS 433, Nature 562, 82 - 85, arXiv:1810.01892

16. Aguilar, M., Ali Cavasonza, L., Alpat, B., et al. (AMS-Collab., includ. W. de Boer, I. Gebauer, E.-S. Seo), (2017), Title: Observation of the Identical Rigidity Dependence of He, C, and O Cosmic Rays at High Rigidities by the Alpha Magnetic Spectrometer on the International Space Station, Phys. Rev. Letters 119, id.251101

17. Aguilar, M., Ali Cavasonza, L., Ambrosi, G., et al. (AMS-Collab., includ. W. de Boer, I. Gebauer, E.-S. Seo), (2018a), Title: Observation of New Properties of Secondary Cosmic Rays Lithium, Beryllium, and Boron by the Alpha Magnetic Spectrometer on the International Space Station, Phys. Rev. Letters 120, id.021101

18. Aguilar, M., Ali Cavasonza, L., Alpat, B., et al. (AMS-Coll., includ. W. de Boer, I. Gebauer, E.-S. Seo), (2018b), Title: Precision Measurement of Cosmic-Ray Nitrogen and its Primary and Secondary Components with the Alpha Magnetic Spectrometer on the International Space Station, Phys. Rev. Letters 121, id.051103

19. Aharonian, F.A., Very high energy cosmic gamma radiation : a crucial window on the extreme Universe (book), World Scientific Publishing, Singapore (2004)

20. Albert, A., André M., Anghinolfi, M., et al. (Antares-Collab., includ. L. Caramete), (2017a), Title: Search for high-energy neutrinos from bright GRBs with ANTARES, Month. Not. Roy. Astr. Soc. 469, 906 - 915

21. Albert, A., André, M., Anghinolfi, M., et al. (ANTARES-Collab., includ. L. Caramete), IceCube-Collab. (includ. J. Becker Tjus and T. Stanev), Pierre Auger-Collab. (includ. P.L. Biermann), LIGO Scientific Collab. (includ. L. Gergely), Virgo Collaboration, (2017b), Title: Search for High-energy Neutrinos from Binary Neutron Star Merger GW170817 with ANTARES, IceCube, and the Pierre Auger Observatory, Astrophys. J. Letters 850, id.L35, eprint arXiv:1710.05839

22. Albert, A., André, M., Anghinolfi, M., et al. (ANTARES-Coll. and IceCube-Coll., includ. L. Caramete, J. Becker Tjus, T. Stanev), (2018), Title: Joint constraints on Galactic diffuse neutrino emission from ANTARES and IceCube, eprint arXiv:1808.03531

23. Alfaro, R., Alvarez, C., Álvarez, J. D., et al. (HAWC-Coll., includ. S. Casanova), (2017), Title: All-particle cosmic ray energy spectrum measured by the HAWC experiment from 10 to $500 \mathrm{TeV}$, Phys. Rev. D 96, id.122001

24. Aliu, E., Archambault, S., Aune, T., et al., (2014), Title: Investigating the TeV Morphology of MGRO J1908+06 with VERITAS, Astrophys. J. 787, id.166

25. Allen, M.L., Ph.D. Thesis, Univ. of Toronto (1999), Title: Radio continuum studies of the evolved starburst in M82

26. Allen, M.L., \& Kronberg, P.P., (1998), Title: Radio Spectra of Selected Compact Sources in the Nucleus of M82, Astrophys. J. 502, 218 - 228 
27. Amato, E., \& Blasi, P., (2017), Title: Cosmic Ray Transport in the Galaxy: a Review, in press in Advances in Sp. Res., eprint arXiv:1704.05696

28. Axford, W.I., Leer, E., \& Skadron, G., (1977), Title: The acceleration of cosmic rays by shock waves, in Proc. 15th Intern. C. R. Conf., Plovdiv, Bulgaria, 11, 132 - 137

29. Baade, W., \& Zwicky, F., (1934), Title: Cosmic rays from supernovae, Proc. Nat. Acad. Sci. 20, 259

30. Bartel, N., Ratner, M.I., Rogers, A.E.E., et al., (1987), Title: VLBI observations of 23 hot spots in the starburst galaxy M82, Astrophys. J. 323, 505 - 515

31. Beck, R., Brandenburg, A., Moss, D., et al., (1996), Title: Galactic Magnetism: Recent Developments and perspectives, Annual Rev. of Astron. E Astrophys. 34, 155 - 206

32. Bell, A.R., (1978a), Title: The acceleration of cosmic rays in shock fronts. I, Month. Not. Roy. Astr. Soc. 182, $147-156$

33. Bell, A.R., (1978b), Title: The acceleration of cosmic rays in shock fronts. II, Month. Not. Roy. Astr. Soc. 182, $443-455$

34. Bethe, H.A., (1990), Title: Supernova mechanisms, Rev. Mod. Phys. 62, 801 - 866

35. Biermann, P.L., \& Strittmatter, P.A., (1987), Title: Synchrotron emission from shockwaves in active galactic nuclei, Astrophys. J. 322, $643-649$

36. Biermann, P.L., Chini, R., Haslam, C.G.T., et al., (1992), Title: Evidence for heavy element dust clumps from the increasing 1300 micron emission from supernova 1987 A, Astron. E Astroph. Letters 255, L5 - L8

37. Biermann, P.L., (1993), referred to as CR-I, Title: Cosmic rays I. The cosmic ray spectrum between $10^{4} \mathrm{GeV}$ and $310^{9} \mathrm{GeV}$, Astron. E Astroph. 271, 649 - 661

38. Biermann, P.L., \& Cassinelli, J.P., (1993), referred to as CR-II, Title: Cosmic rays II. Evidence for a magnetic rotator Wolf-Rayet star origin, Astron. \& Astroph. 277, 691 - 706

39. Biermann, P.L., \& Strom, R.G., (1993), referred to as CR-III, Title: Cosmic Rays III. The cosmic ray spectrum between $1 \mathrm{GeV}$ and $10^{4} \mathrm{GeV}$ and the radio emission from supernova remnants, Astron. $\mathcal{E}$ Astroph. 275, 659 669

40. Biermann, P.L., Langer, N., Seo, E.-S., et al., (2001), Title: Cosmic Rays IX. Interactions and transport of cosmic rays in the Galaxy, Astron. \& Astroph. 369, 269 - 277

41. Biermann, P.L., Becker, J.K., Meli, A., et al., (2009), Title: Cosmic Ray Electrons and Positrons from Supernova Explosions of Massive Stars, Phys. Rev. Letters 103, id.061101, arXiv:0903.4048,

42. Biermann, P.L., Caramete, L.I., Fraschetti, F., et al. (includ. L.Á. Gergely, A. Meli, B.B. Nath, \& J. Becker Tjus), (2016), Title: The nature and origin of ultra-high energy cosmic ray particles, review at the Vulcano meeting, Vulcano Island, May 2016, http:/ / www.lnf.infn.it/sis/frascatiseries/Volume64/Volume64.pdf, arXiv:1610.00944

43. Biermann, P.L., Becker Tjus, J., de Boer, W., et al., (includ. P.P. Kronberg, A. Meli, B.B. Nath, T. Stanev), (2018), referred to as ASR18, Title: Supernova explosions of massive stars and cosmic rays, Advances in Space Research 62, 2773 - 2816, https:/ / doi.org/10.1016/j.asr.2018.03.028, eprint arXiv:1803.10752

44. Bisnovatyi-Kogan, G.S., (1970), Title: The Explosion of a Rotating Star As a Supernova Mechanism, Astron. Zh. 47, 813 - 816, transl. Sov. A.J. 14, 652 - 655 (1971);

45. Bisnovatyi-Kogan, G.S., Moiseenko, S.G., \& Ardelyan, N.V., (2008), Title: Different magneto-rotational supernovae, Astron. Rep. 52, 997 - 1008

46. Blandford, R.D., \& Ostriker, J.P., (1978), Title: Particle acceleration by astrophysical shocks, Astrophys. J. Letters 221, L29 - L32

47. Blasi, P., (2013), Title: The origin of galactic cosmic rays, Astron. E Astroph. Rev. 21, id.70

48. de Boer, W., Bosse, L., Gebauer, I., et al. (includ. P.L. Biermann), (2017), Title: Molecular Clouds as the Origin of the Fermi Gamma-Ray GeV-Excess, Phys. Rev. D 96, id.043012, arXiv:1707.08653

49. Bykov, A.M., Ellison, D.C., Gladilin, P.E., et al., Proc. HIGH ENERGY GAMMA-RAY ASTRONOMY: 5th International Meeting on High Energy Gamma-Ray Astronomy. (2012), Title: Galactic cosmic ray origin sites: Supernova remnants and superbubbles, AIP Conf. Proc. 1505, 46 - 55

50. Bykov, A.M., Ellison, D.C., Marcowith, A., et al., (2018), Title: Cosmic Ray Production in Supernovae, Sp. Sci. Rev. 214, id.41

51. Caprioli, D., (2012), Title: Fermi acceleration at supernova remnant shocks, in AIP Conf. Proc. 1505, 237 - 240

52. Caramete, L.I., \& Biermann, P.L., (2010), Title: The mass function of nearby black hole candidates, Astron. $\mathcal{E}$ Astroph. 521, id.A55, ; arXiv:0908.2764 
53. Chevalier, R.A., (1982), Title: Are young supernova remnants interacting with circumstellar gas?, Astrophys. J. Letters 259, L85 - L89

54. Chini, R., Hoffmeister, V.H., Nasseri, A., et al., (2012), Title: A spectroscopic survey on the multiplicity of high-mass stars, Month. Not. Roy. Astr. Soc. 424, 1925 - 1929

55. Chini, R., Nasseri, A., Dembsky, T., et al., (2013), Title: Stellar multiplicity across the mass spectrum, in Setting a New Standard in the Analysis of Binary Stars, Edited by K. Pavlovski, A. Tkachenko \& G. Torres. EAS Publ. Ser., 64, 155 - 162

56. Colgate, S.A., (2004), Title: Acceleration mechanisms 2: force-free reconnection, Comptes Rendus Physique 5, No.4, 431 - 440

57. Colgate, S.A., Fowler, T.K., Li, H., et al., (2014), Title: Quasi-Static Model of Collimated Jets I. Accretion Disk and Jets, ApJ 789, id.144

58. Cox, D.P., (1972), Title: Cooling and Evolution of a Supernova Remnant, Astrophys. J. 178, 159 - 168

59. Dickel, J.R., van Breugel, W.J.M., \& Strom, R.G., (1991), Title: Radio structure of the remnant of Tycho's supernova (SN 1572), Astron. J. 101, 2151 - 2159

60. Diehl, R., (2013), Title: Nuclear astrophysics lessons from INTEGRAL, Rep. Pro.Phys. 76, id.026301

61. Diehl, R., (2017), Title: Gamma-ray line measurements from supernova explosions, in Proc. IAU Symposium 331 "SN1987A 30 years after", La Reunion, Feb. 2017. Accepted for publication in IAU Conf Proc., eprint arXiv:1704.05937

62. Drury, L.O'C, (1983), Title: An introduction to the theory of diffusive shock acceleration of energetic particles in tenuous plasmas, Rep. Pro.Phys. 46, 973 - 1027

63. Fenech, D.M., Muxlow, T.W.B., Beswick, R.J., et al., (2008), Title: Deep MERLIN 5GHz radio imaging of supernova remnants in the M82 starburst, Month. Not. Roy. Astr. Soc. 391, 1384 - 1402

64. Fenech, D.M., Beswick, R.J., Muxlow T.W.B., et al., (2010), Title: Wide-field Global VLBI and MERLIN combined monitoring of supernova remnants in M82, Month. Not. Roy. Astr. Soc. 408, 607 - 621

65. Fermi, E., (1949); Title: On the Origin of the Cosmic Radiation, Phys. Rev. 75, 1169 - 1174

66. Fermi, E., (1954); Title: Galactic Magnetic Fields and the Origin of Cosmic Radiation, Astrophys. J. 119, 1 - 6

67. Fischer, T., Bastian, N.-U.F., Wu, M.-R., et al., (2018), Title: Quark deconfinement as a supernova explosion engine for massive blue supergiant stars, Nature Astron, doi.org/10.1038/s41550-018-0583-0

68. Gaisser, T.K., Engel, R., \& Resconi, E., Cosmic Rays and Particle Physics (book), Cambridge, UK, Cambridge University Press (2016)

69. Gendre, M.A., Fenech, D.M., Beswick, R.J., et al., (2013), Title: Flux density variations of radio sources in M82 over the last three decades, Month. Not. Roy. Astr. Soc. 431, $1107-1120$

70. Gergely L.Á., \& Biermann, P.L., (2009), Title: Supermassive black hole mergers, Astrophys. J. 697, 1621 - 1633 , arXiv:0704.1968

71. Ginzburg, V. L., \& Syrovatskii, S. I., (1963), Title: Cosmic Rays in Metagalactic Space, Astron. Zh. 40, 466, transl. in Sov. Astron. A.J. 7, 357 (1963a)

72. Ginzburg, V.L., \& Ptuskin, V.S., (1976), Title: On the origin of cosmic rays: some problems in high-energy astrophysics, Rev. Mod. Phys. 48, 161 - 189

73. Golla, G., Allen, M.L., \& Kronberg, P.P., (1996), Title: The Starburst Nuclear Region in M82 Compared in Several Wave Bands, Astrophys. J. 473, 244 - 253

74. Halzen, F., lectures at Erice (2018): under https:/ /icecube.wisc.edu/ halzen/presentations.htm

75. Hamuy, M., (2003), Title: Observed and Physical Properties of Core-Collapse Supernovae, Astrophys. J. 582, 905 - 914

76. Heesen, V., Krause, M., Beck, R., et al., (2018), Title: Radio haloes in nearby galaxies modelled with 1D cosmic ray transport using SPINNAKER, Month. Not. Roy. Astr. Soc. 476, 158 - 183

77. Heinz, S., \& Sunyaev, R., (2002), Title: Cosmic rays from microquasars: A narrow component to the CR spectrum?, Astron. \& Astroph. 390, 751 - 766

78. Hess, V.F., (1912), Title: Die Beobachtungen der durchdringenden Strahlung bei sieben Freiballonfahrten, Physik. Z. 13, 1084

79. https://www.mpi-hd.mpg.de/hfm/HESS/pages/home/som/2017/09/ (2018)

80. Hillas, A.M., (1984), Title: The Origin of Ultra-High-Energy Cosmic Rays, Annual Rev. of Astron. E Astrophys. 22, $425-444$ 
81. Jokipii, J.R., \& Morfill, G., (1987), Title: Ultra-high-energy cosmic rays in a galactic wind and its termination shock, Astrophys. J. 312, 170 - 177

82. Jokipii, J.R., (1987), Title: Rate of energy gain and maximum energy in diffusive shock acceleration, Astrophys. J. 313, 842 - 846

83. Kadler, M., Krauß, F., Mannheim, K., et al., (2016), Title: Coincidence of a high-fluence blazar outburst with a PeV-energy neutrino event, Nature Phys. 12, 807 - 814

84. Kardashev, N.S., (1962), Title: Nonstationarity of Spectra of Young Sources of Nonthermal Radio Emission, Astron. Zh. 39, 393 - 409, transl. Sov. Astron. A.J. 6, 317 - 327 (1962)

85. Krause, M., Irwin, J., Wiegert, Th., et al., (2018), Title: CHANG-ES. IX. Radio scale heights and scale lengths of a consistent sample of 13 spiral galaxies seen edge-on and their correlations, Astron. E Astroph. 611, id.A72

86. Kohlhörster, W., (1913), Title: Messungen der durchdringenden Strahlung im Freiballon in größeren Höhen, Physik. Z. 14, 1153

87. Kotera, K., Olinto, A.V., (2011), Title: The Astrophysics of Ultrahigh-Energy Cosmic Rays, Annual Rev. of Astron. E Astrophys. 49, 119 - 153

88. Kronberg, P.P., \& Wilkinson, P.N., (1975), Title: High-resolution, multifrequency radio observations of M82, Astrophys. J. 200, 430 - 435

89. Kronberg, P.P., Biermann, P.L., \& Schwab, F.R., (1981), Title: The continuum radio structure of the nucleus of M82, Astrophys. J. 246, 751 - 760

90. Kronberg, P.P., Biermann, P.L., \& Schwab, F.R., (1985), (referred to as KBS85) Title: The nucleus of M82 at radio and X-ray bands - Discovery of a new radio population of supernova candidates, Astrophys. J. 291, 693 - 707

91. Kronberg, P.P., Sramek, R.A., Birk, G.T., et al., (2000), Title: A Search for Flux Density Variations in 24 Compact Radio Sources in M82, Astrophys. J. 535, 706 - 711

92. Kronberg, P.P., Dufton, Q.W., Li, H., et al., (2001), Title: Magnetic energy of the Intergalactic Medium from galactic black holes, ApJ 560, 178 - 186

93. Kronberg, P.P., (2002), Title: Intergalactic Magnetic Fields, Phys. Today (invited feature article) 55, No.12, 40 46

94. Kronberg, P.P., Cosmic Magnetic Fields (book), Cambridge University Press, Cambridge (2016)

95. Krymskii, G.F., (1977), Title: A regular mechanism for the acceleration of charged particles on the front of a shock wave, Akad. Nauk Dokl. 234, 1306 - 1308, transl. Sov. Phys. - Dokl. 22, 327 - 328 (1977);

96. Kun, E., Biermann, P.L., \& Gergely, L.Á., (2017), Title: A flat spectrum candidate for a track-type high energy neutrino emission event, the case of blazar PKS 0723-008, Month. Not. Roy. Astr. Soc. Lett. 466, L34 - L38

97. Kun, E., Biermann, P.L., Gergely, L.Á. , (2018), Title: VLBI radio structure and core-brightening of the high-energy neutrino emitter TXS 0506+056, submitted to Month. Not. Roy. Astr. Soc. Lett., eprint arXiv:1807.07942

98. Landau, L.D., \& Lifshitz, E.M., Fluid mechanics, Course of theoretical physics (book series), Oxford: Pergamon Press, (1959); translated from Russian

99. Letessier-Selvon, A., \& Stanev, T., (2011), Title: Ultrahigh energy cosmic rays, Rev. Mod. Phys. 83, 907 - 942

100. Linsley, J., (1963), Title: Evidence for a Primary Cosmic-Ray Particle with Energy $10^{20} \mathrm{eV}$, Phys. Rev. Letters 10, 146 - 148

101. Lovelace, R.V.E., (1976), Title: Dynamo model of double radio sources, Nature 262, 649 - 652

102. Lusk, J.A., \& Baron, E., (2017), Title: Bolometric Light Curves of Peculiar Type II-P Supernovae, Publ. Astron. Soc. Pac. 129, p.044202

103. Maeda, K., Tominaga, N., Umeda, H., et al., (2010), Title: Supernova nucleosynthesis and stellar population in the early Universe, Mem. Soc. Astron. Ital. 81, 151 - 156

104. McDonald, A.R., Muxlow, T.W.B., Wills, K.A., et al., (2002), A parsec-scale study of the 5/15-GHz spectral indices of the compact radio sources in M82, Month. Not. Roy. Astr. Soc. 334, $912-924$

105. Meli, A., \& Biermann, P.L., (2006), Title: Cosmic rays X. The cosmic ray knee and beyond: diffusive acceleration at oblique shocks, Astron. E Astroph. 454, 687 - 694

106. Meli, A., Becker, J. K., \& Quenby, J. J., (2008), Title: On the origin of ultra high energy cosmic rays: subluminal and superluminal relativistic shocks, Astron. E Astroph. 492, 323 - 326

107. Merten, L., Bustard, Ch., Zweibel, E.G., et al., (2018), Title: The Propagation of Cosmic Rays from the Galactic Wind Termination Shock: Back to the Galaxy?, Astrophys. J. 859, id.63 
108. Milgrom, M., \& Usov, V., (1995), Title: Possible Association of Ultra-High-Energy Cosmic-Ray Events with Strong Gamma-Ray Bursts, Astrophys. J. Letters 449, L37 - L40

109. Milgrom, M., \& Usov, V., (1996), Title: Gamma-ray bursters as sources of cosmic rays, Astropart. Phys. 4, 365 369

110. Mirabel, I.F., (2004), Title: Microquasars and ULXs: Fossils of GRB Sources, in Proc. Compact Binaries in the Galaxy and Beyond, Eds. G. Tovmassian and E. Sion. Rev. Mex. de Astron. Astrof. (Serie de Conf.) Vol. 20. IAU Colloquium 194, p. 14 - 17 (http:/ / www.astroscu.unam.mx/ rmaa/)

111. Mirabel, I.F., (2011), Title: Microquasars, Mem. Soc. Astron. Ital. 82, 14 - 23

112. Mirabel, I. F., (2017a), Title: The Formation of Stellar Black Holes, in New Astronomy Reviews; doi:10.1016/j.newar.2017.04.002, eprint arXiv:1609.08411,

113. Mirabel, I. F., (2017b), Title: Stellar progenitors of black holes: insights from optical and infrared observations, in New Frontiers in Black Hole Astrophysics, Proc. of the IAU, IAU Symposium, Volume 324, 27 - 30

114. Mirabel, I. F., (2017c), Title: Black holes formed by direct collapse: observational evidences, in New Frontiers in Black Hole Astrophysics, Proc. of the IAU, IAU Symposium, Volume 324, 303 - 306

115. Miralda-Escudé, J., \& Waxman, E., (1996), Title: Signatures of the Origin of High-Energy Cosmic Rays in Cosmological Gamma-Ray Bursts, Astrophys. J. Letters 462, L59 - L62

116. Murphy, R.P., Sasaki, M., Binns, W.R., et al., (2016), Title: Galactic Cosmic Ray Origins and OB Associations: Evidence from SuperTIGER Observations of Elements 26Fe through 40Zr, Astrophys. J. 831, id.148, arXiv:1608.08183

117. Muxlow, T.W.B., Pedlar, A., Wilkinson, P.N., et al., (1994), Title: The structure of young supernova remnants in M82, Month. Not. Roy. Astr. Soc. 266, $455-467$

118. Muxlow, T.W.B., Pedlar, A., Beswick, R.J., et al., (2005), Title: Is $41.95+575$ in M82 actually an SNR?, Mem. S.A.It. 76, $586-588$

119. Nakar, E., Poznanski, D., Katz, B., (2016); Title: The Importance of ${ }^{56} \mathrm{Ni}$ in Shaping the Light Curves of Type II Supernovae, Astrophys. J. 823, id.127

120. Nayerhoda, A., Salesa Greus, F., \& Casanova, S., for the HAWC coll., (2018), Title: TeV Diffuse emission from the inner Galaxy, Front. Astron. E Sp. Sci. 5, id.8

121. Parker, E.N., (1958), Title: Dynamics of the Interplanetary Gas and Magnetic Fields, Astrophys. J. 128, 664 676

122. Piran, T., (2004), Title: The physics of gamma-ray bursts, Rev. Mod. Phys. 76, 1143 - 1210

123. Raddi, R., Hollands, M.A., Gänsicke, B.T., et al., (2018), Title: Anatomy of the hyper-runaway star LP 40-365 with Gaia, Month. Not. Roy. Astr. Soc. Lett. 479, L96 - L101

124. Rossa, J., \& Dettmar, R.-J., (2003), Title: An H $\alpha$ survey aiming at the detection of extraplanar diffuse ionized gas in halos of edge-on spiral galaxies. I. How common are gaseous halos among non-starburst galaxies?, Astron. E Astroph. 406, 493 - 503

125. Sedov, L. I., (1958), Title: Examples of Gas Motion and Certain Hypotheses on the Mechanism of Stellar Outbursts, Rev. Mod. Phys. 30, 1077 - 1079

126. Seo, E.-S., et al., (2014), Title: Cosmic Ray Energetics And Mass for the International Space Station (ISS-CREAM), Adv. in Sp. Res., 53/10, 1451 - 1455

127. Seo, E.-S., (2018), https://indico.desy.de/indico/event/18204/session/8/contribution/376/material/slides/2.pdf

128. Seo, J., Kang, H., Ryu, D., (2018), Title: The Contribution of Stellar Winds to Cosmic Ray Production, J. Korean Astron. Soc. 51, $37-48$

129. Shen, K.J., Kasen, D., Miles, B.J., et al., (2018a), Title: Sub-Chandrasekhar-mass White Dwarf Detonations Revisited, Astrophys. J. 854, id.52

130. Shen, K.J., Boubert, D., Gänsicke, B.T., et al., (2018b), Title: Three Hypervelocity White Dwarfs in Gaia DR2: Evidence for Dynamically Driven Double-degenerate Double-detonation Type Ia Supernovae, Astrophys. J. 865 , id.15

131. Spitzer, L. Jr., (1962), Physics of Fully Ionized Gases (book), second Ed., Wiley Interscience, New York

132. Stanev, T., Biermann, P.L., \& Gaisser, T.K., (1993); referred to as CR-IV, Title: Cosmic rays IV. The spectrum and chemical composition above $10^{4} \mathrm{GeV}$, Astron. E Astroph. 274, 902 - 908

133. Stanev, T., High Energy Cosmic Rays (book), Springer, Heidelberg (2010) 
134. Utrobin, V.P., Wongwathanarat, A., Janka, H.-Th., et al., (2017), Title: Light-curve Analysis of Ordinary Type IIP Supernovae Based on Neutrino-driven Explosion Simulations in Three Dimensions, Astrophys. J. 846, id. 37

135. Vietri, M., (1995), Title: The Acceleration of Ultra-High-Energy Cosmic Rays in Gamma-Ray Bursts, Astrophys. J. 453, $883-889$

136. Vietri, M., Month. Not. Roy. Astr. Soc. Lett. 278, L1 - L4 (1996); Title: Coronal gamma-ray bursts as the sources of ultra-high-energy cosmic rays?

137. Vietri, M., (1998), Title: Ultrahigh Energy Neutrinos from Gamma Ray Bursts, Phys. Rev. Letters 80, 3690 3693

138. Völk, H.J., \& Biermann, P.L., (1988), Title: Maximum energy of cosmic-ray particles accelerated by supernova remnant shocks in stellar wind cavities, Astrophys. J. Letters 333, L65 - L68

139. Vulic, N., Hornschemeier, A.E., Wik, D.R., et al., (2018), Title: Black Holes and Neutron Stars in Nearby Galaxies: Insights from NuSTAR, Astrophys. J. , in press, arXiv:1808.05617,

140. Waxman, E., (1995), Title: Cosmological Gamma-Ray Bursts and the Highest Energy Cosmic Rays, Phys. Rev. Letters 75, $386-389$

141. Waxman, E., \& Bahcall, J., (1997), Title: High Energy Neutrinos from Cosmological Gamma-Ray Burst Fireballs, Phys. Rev. Letters 78, 2292 - 2295

142. Weber, E.J., \& Davis, L.Jr., (1967); Title: The Angular Momentum of the Solar Wind, Astrophys. J. 148, 217 227

143. Weliachew, L., Fomalont, E.B., \& Greisen, E.W., (1984), Title: Radio observations of H I and OH in the center of the galaxy M82, Astron. E Astroph. 137, 335 - 342

144. Wesson, R., Barlow, M.J., Matsuura, M., et al., (2015), Title: The timing and location of dust formation in the remnant of SN 1987A, Month. Not. Roy. Astr. Soc. 446, 2089 - 2101

145. Yüksel, H., Kistler, M.D., Stanev, T., (2009), Title: TeV Gamma Rays from Geminga and the Origin of the GeV Positron Excess, Phys. Rev. Letters 103, id.051101

146. Zhang, B., \& Mészáros, P., (2004), Title: Gamma-Ray Bursts: progress, problems \& prospects, Int. J. Mod. Phys. A 19, $2385-2472$ 